\title{
Nurse educators' perceptions of their own self-leadership: An explorative qualitative inquiry
}

\author{
Vhothusa Edward Matahela* \\ National Department of Health, South Africa
}

Received: July 19, 2021

Accepted: August 18, 2021

Online Published: September 17, 2021

DOI: $10.5430 /$ jnep.v12n2p1

URL: https://doi.org/10.5430/jnep.v12n2p1

\begin{abstract}
Background and objective: Organisations, including academic institutions, are moving away from the traditional top-down command and control leadership styles to a focus on internal leadership (self-leadership) of staff. In the absence of traditional external leadership, individuals with self-leadership skills and qualities can make smart decisions to achieve organisational success. Despite the availability of literature studies on nurses' self-leadership, there is a dearth of studies addressing self-leadership in nurse educators. It is imperative that a study exploring nurse educators' perceptions regarding their self-leadership be conducted to achieve a comprehensive understanding of the phenomenon. This would assist in identifying the role that stakeholders play in creating an enabling academic environment that promotes the development, the practice, and supportive interventions of self-leadership. This study sought to explore and describe the perceptions of nurse educators regarding their self-leadership and how this can be facilitated in a nursing education institution (NEI) context.

Methods: A qualitative, exploratory, and descriptive research design was used to provide an in-depth exploration of the perceptions of nurse educators regarding their self-leadership in an NEI setting. Four semi-structured focus group interviews were conducted with purposively selected nurse educators, and audiotaped until data saturation resulted. The researcher and an independent coder analysed the data using Tesch protocols on thematic analysis. Lincoln and Guba's strategies were used to achieve trustworthiness.

Results: Two themes emerged, namely: nurse educators' perceptions of self-leadership; and the facilitation of self-leadership in nurse educators.

Conclusions: The nurse educators mirrored themselves as leaders in action and are aware of the importance of their engagement in self-leadership activities in learning and teaching. Facilitating self-leadership within nurse educators was perceived to be the responsibility of individual nurse educators, and to a certain extent their colleagues, and that of the institutional management team.
\end{abstract}

Key Words: Leadership, Motivation, Nurse educator, Nursing education institution, Reflection, Self-leadership, South Africa

\section{INTRODUCTION}

In order to ensure that South Africa keeps abreast of technological change, globalisation, and modernisation, the South African government has introduced numerous policy frameworks aimed at improving service delivery quality in all sectors, including the health sector. The frameworks include the National Development Plan, the blueprint document that calls for health systems' strengthening of health establishments and institutions by filling vacant posts with skilled, committed, and competent health professionals by $2030 .^{[1]}$ The country is currently undergoing legislative reforms that require nursing education to be positioned in the higher education band, and this will influence training of nurses in the country. ${ }^{[2]}$ Thus, there is a call for a new way of leadership

*Correspondence: Vhothusa Edward Matahela; Email: vhothusa@yahoo.com; Address: National Department of Health, South Africa. 
in NEIs that ensures organisational success during the implementation of these reforms, whilst simultaneously improving the country's health systems' functioning. ${ }^{[3]}$

Due to the significant problem of increasing unprofessional behaviour and conduct by public servants, including healthcare workers, the South African government introduced the Public Service Charter to curtail poor leadership, maladministration, and misconduct of public servants. ${ }^{[4]}$ The Charter aims to commit public servants to being polite, helpful, and accessible to the public, and to treat them as customers entitled to high service standards. Public servants, including nurse educators, are also expected to work within the policy framework of the Batho Pele (putting people first) principles, a government initiative to encourage public servants to be service-orientated, and to strive for excellence in service delivery and commit themselves to continuous service delivery improvement. ${ }^{[5]}$

Although nurse education is a rewarding and fulfilling career, nurse educators may also find themselves involved in undesirable situations that negatively affect the teaching-learning environment. According to Clark, ${ }^{[6]}$ in classroom situations nurse educators sometimes experience disruptive and uncivil student behaviour, which can lower self-esteem and create self-doubt in the educator. However, as leaders, nurse educators are expected to deal with such situations with a positive state of mind, exercising optimal self-esteem, confidence, calm, thoughtfulness, reflection, and resilience, in order to model and promote students' professional development. Moreover, educators are expected to apply a positive moral perspective to lead by example during verbal communication, and demonstrate high moral standards and values that contribute to supportive learning environments. ${ }^{[7]}$

Thus, it is incumbent upon nurse educators to conduct themselves in a manner that demonstrates self-leadership through self-control, self-regulation, and professional modelling to ensure that the classroom is a conducive and non-threatening learning and teaching environment.

In today's organisations, the emergence of self-leadership as a means of achieving organisational success is partly due to an increased need for self-directed and self-motivated employees to reduce the bureaucratic work structures and to ease the designated leaders' workload. ${ }^{[8]}$ Jooste $^{[9]}$ describes self-leadership as the ways in which individuals influence themselves to improve their performance. Stewart and colleagues ${ }^{[10]}$ assert that self-leadership is driven by external factors that influence leadership practice, such as the empowering leader in an institution, and internal forces that originate from the individual self, who ultimately controls their actions taken in a particular context. Thus, although self-leadership is an individual's internal process, it is the responsibility of both the individual and the organisation to create the conditions necessary to motivate individuals to become achievement-oriented goal-setters and initiators. ${ }^{[11]}$ For the purpose of this study, self-leadership takes place when nurse educators self-direct and motivate themselves to succeed and improve performance in an NEI setting. ${ }^{[8]}$

As leaders, nurse educators are valuable assets in their respective institutions, and as such, are strongly expected to mirror their institution's core values in the myriad and diverse tasks in which they engage. ${ }^{[12]}$ Leadership experts such as Neck and colleagues ${ }^{[13]}$ propose that one cannot be expected to lead others effectively if they are unable to lead themselves effectively. The challenges that nurse educators encounter during learning and teaching require nurse educators to be creative, innovative, resilient, and self-leaders. Through selfleadership, nurse educators should be able to role model accepting responsibility for their behaviours and outcomes to colleagues and students. ${ }^{[9]}$ Although self-leadership has been widely researched in other disciplines such as sports, teaching, business and management, Ntshingila et al's ${ }^{[14]}$ study established that the self-leadership concept is poorly understood in nursing, and propose that the concept's meaning and values require further investigation. Matahela' ${ }^{[15]}$ integrative literature review established that the meaning of self-leadership in an NEI context was not clearly elucidated, and recommend that further concept exploration and description, from nurse educators themselves in an NEI setting should be conducted, using exploratory methods including interviews.

The researcher is concerned that if student nurses are trained by educators who do not demonstrate self-leadership and role model good behaviour, values, and attitudes, government's health reform programmes will not be realised, because students learn behaviours during their socialisation process into nursing during the learning and teaching process. If nurse educators engage in self-leadership practices, this can easily be inculcated into students, resulting in the production of nurses with self-leadership dispositions necessary to produce quality patient care.

The central questions that guided the study are:

- How do you perceive your self-leadership?

- What can be done to facilitate self-leadership within nurse educators in NEIs?

\section{Methodology}

\subsection{Design}

A qualitative, exploratory, and descriptive research design was used to conduct semi-structured focus group interviews. 
Qualitative research provided the researcher with an opportunity to explore and describe in depth the nurse educators' perceptions regarding their own self-leadership in NEI settings.

\subsection{Population and sampling}

The population were nurse educators teaching in purposively selected NEIs. The participants had to be teaching on a fulltime basis for at least one year and had to be readily available, for inclusion in the study. Although self-leadership practices improve overtime, a years' experience in an NEI would be adequate for the participants to develop an informed perception of their experiences of their own self-leadership in their work setting. ${ }^{[16]}$ Table 1 displays the demographic variations of the participants.

\subsection{Data collection}

The qualitative data was collected using four focus group interviews which comprised of seven to nine nurse educators each, who were purposively selected. The focus group interviews were conducted by the researcher, who has vast experience in nursing education instruction and leadership, has a master's degree in nursing education and is well experienced in qualitative research. The interviews were conducted between January and December 2017, and English was the medium of engagement. The interviews lasted for an average period of 45-60 minutes each. Data saturation guided the number of focus groups. All the interviews were conducted in nurse educators' natural settings, that is, in the institutions where the nurse educators work, with permission being granted by the relevant authorities and participants. An interview guide with possible probing questions was shared with participants prior to interviews. The guide was used during interviews, however, participants' responses were guided by probing and clarifying their answers. The researcher used other communication skills such as reflecting, remaining silent, and nodding, to obtain as much information on the research questions as possible. All interviews were audiotaped with the participants' permission. The researcher took field notes of non-verbal cues and nuances during the interviews to support data collection. Each interview was transcribed verbatim as soon as the interview was concluded, enabling the researcher to become familiar and immersed in the data as he listened to the audiotapes and re-read the transcripts and field notes.

\subsection{Data analysis}

The researcher transcribed each interview verbatim as soon as the interview had been conducted. This enabled the researcher to become familiar with the data as he listened to the tapes, re-read the transcripts and field notes, and thus became Published by Sciedu Press immersed in the data. The researcher manually analysed the verbatim transcripts and field notes obtained from the semi-structured focus group interviews according to Tesch's protocol. ${ }^{[17]}$ This entailed reading and re-reading all the transcripts carefully to get a sense of the whole to be familiar with data. Thereafter initial codes were generated according to themes and subthemes, reviewing themes, and defining and naming themes.

Verbatim transcripts were also sent to an independent coder, a nurse educator with extensive experience in qualitative studies, to analyse the data independently in accordance with the Tesch protocols. A consensus meeting was held between the researcher and the independent coder for discussions on themes and subthemes, verifying if patterns and themes were similar and how they would be displayed in a meaningful and useful manner. To ensure that the themes reflected the participants' voices, the researcher and the co-coder revisited the names of all themes and ensured that the words of participants were used in the names. ${ }^{[18]}$ The audiotapes, printed transcripts and field notes were kept in a safe place under lock and key. The data analysis results revealed two main themes, namely, nurse educators' self-perceptions of self-leadership, and facilitation of self-leadership in nurse educators.

\subsection{Trustworthiness}

Lincoln and Guba's ${ }^{[19]}$ criteria of trustworthiness, which include credibility, transferability, dependability, and confirmability, were applied to ensure rigour. Credibility was ensured by presenting data on nurse educators' self-leadership accurately, so that other nurse educators who had experienced similar experiences would immediately recognise the descriptions. This was achieved through: prolonged engagement by spending sufficient time with the participants prior to and during the interviews; triangulation, through using multiple data collection methods and taking field notes; and member-checking, which involved returning to participants and verifying that the transcribed data was a truthful rendition of their experience. Providing a comprehensive description of the study methods ensured transferability. Dependability was maintained by providing a comprehensive description of the research methodology, and confirmability was ensured by means of an audit trail, triangulation, and consensus discussions between the researcher and the independent coder.

\subsection{Ethical considerations}

Ethical clearance was sought from the university's Research and Ethics Committee (REC-012714-039) before the study was conducted. Permission was also sought from the relevant research structures and authorities. After the researcher provided a thorough explanation of the purpose, objectives, and 
methodology of the study, all participants voluntarily participated in the study by signing an informed consent form. Participants were also informed of their right to withdraw from the study at any time without any consequences.

The following ethical standards for nurse researchers were upheld as outlined by Brink and colleagues: ${ }^{[20]}$ fostering of justice; beneficence; excluding harm and exploitation of participants; self-determination; ensuring confidentiality and anonymity; and ensuring quality research.

\section{Results}

All the participants were female, with the youngest at 25 and the oldest at 60 years of age. The participants were involved in a myriad of activities in the NEI such as facilitation of theory, clinical teaching, clinical accompaniment, supervision of research, quality coordination, student counselling, and student administration (see Table 1).

Table 1. Demographics of the participants

\begin{tabular}{|l|l|}
\hline Participant demographics & $\mathbf{N}=\mathbf{2 6}$ \\
\hline Gender & 26 Females, 0 Males \\
\hline Age range & 25-60 years old \\
\hline Race & 17 African, 6 Coloured, 3 White \\
\hline Highest Qualification & 3 Doctorate, 10 Master's, 6 Postgraduate Diploma, 7 Degree \\
\hline Range of Years of experience in clinical nursing & 3-16 years \\
\hline Range Years of experience in nursing education & 1-21years \\
\hline Number of participants per type of NEI & $\begin{array}{l}\text { 12 Private nursing school, 8 Public nursing college and 6 University-based } \\
\text { nursing department }\end{array}$ \\
\hline Position (title) at NEI & 11 Senior lecturers, 10 Lecturer, 3 Clinical lecturers, 2 Contract lecturers \\
\hline Roles at NEI & $\begin{array}{l}\text { Facilitation of theory, Clinical teaching, Clinical accompaniment, } \\
\text { Supervision of research, Quality coordination, Student counselling, } \\
\text { Student administration }\end{array}$ \\
\hline
\end{tabular}

Two themes emerged from this study, namely nurse educators' perceptions of self-leadership, and the facilitation of self-leadership in nurse educators. These findings are discussed and supported by participants' verbatim statements, and conceptualisation within the existing relevant literature.

\subsection{Theme 1: Nurse educators' perceptions of self- leadership}

In this theme, nurse educators perceived that they engaged in self-leadership practices at their NEIs. Four subthemes emerged: the perception of self-leadership through selfmirroring; the perception of self-leadership in relation to knowledge; the perception of self-leadership in relation to students; and the perception of self-leadership within the community structure.

\subsubsection{Perception of self-leadership through self-mirroring}

The nurse educators perceived self-leadership as selfmirroring, that is, being consciously aware of their own characters, strengths and weaknesses, values, beliefs, and emotions. In mirroring their "selves", nurse educators described their self-leadership as those practices adopted from those whom they admired, such as those they considered role models. This process assisted nurse educators to develop self-confidence, assertiveness, ambition, and commitment to meaningful tasks. In mirroring their "selves", nurse educators described their self-leadership as incorporating visionary leadership, self-motivation, and possessing sound interpersonal skills.

The participants viewed their self-leadership as being able to formulate a vision that articulates their passion for teaching. Such a vision would be aimed at influencing change beyond teaching. One participant said, "As a nurse educator you must be a visionary leader, know your purpose, mission, and understand the people that you are leading-students." Another participant stated, "I cannot lead students if I don't know where I am going."

Being a visionary leader also entailed being a dynamic or futuristic-oriented nurse educator who thinks strategically of where they desire to be in the future. One participant said:

"I can say a leader shouldn't just be focused on what you do here, you should be dynamic, you must be a leader in all spheres, and that also will equip you as a leader, even with specific job that you are doing."

The above statements attest to the need for nurse educators to possess a personal vision to effect change in nursing education. A nurse educator's vision that extends beyond the 
classroom could lead to students' exposures to wider learning experiences.

Participants also viewed self-leadership as being able to find reasons and strength to engage in their tasks despite challenging situations, on their own.

Self-leadership was described as keeping oneself motivated in order to achieve one's set goals, as indicated by this statement:

"I think in self-leadership, you must have self-motivation. You must be motivated to achieve one's goals that you have set about yourself."

A participant described self-leadership as "being motivated even when one is aware of weaknesses." Another participant described how she maintains self-leadership practice through self-talk: "I think on a daily basis you have to talk to yourself and tell yourself to push harder."

The above statements indicate that practicing self-leadership requires nurse educators who are internally motivated to achieve the goals that they set for themselves.

Self-leadership was also viewed as the ability to be introspective regarding the relationship between participants and others. The participants indicated that throughout their experiences they continually reflected, questioned, and re-assessed their values, strengths, and weaknesses. This exercise helped them to better understand their own purposes, beliefs, and emotional states, and to reflect on their teaching abilities and correct performance flaws. One participant said: "it starts with the inner self-organisation; being emotionally mature to want to understand what makes you vulnerable, so that you are able to work on your weaknesses."

The participants viewed their self-leadership as being able to create an atmosphere that promoted good communication and relationships during interactions with students and colleagues, as indicated by the following quotes:

"There are situations when one needs to be affirmative-to be firm and stand your ground and guide students on what is expected of a professional nurse."

"I realise that as a new nurse educator I develop confidence to teach through use of good communication skills."

The participants cited above identified interpersonal skills as the basic skills necessary for effective learning and teaching, as well as fostering collaboration amongst teams.

\subsubsection{Perception of self-leadership in relation to knowl- edge}

The nurse educators perceived their self-leadership as being related to knowledge. According to the participants

Published by Sciedu Press self-leadership in relation to knowledge manifested through acts of self-management, reflective practice, and knowledgeseeking.

The participants described their self-leadership as being in possession of self-management, that is, being better organised and systematic in their work practices. Examples of self-management cited by the participants include timemanagement by being at work and class on time, prioritising their workload, developing lesson plans, meeting deadlines, and adhering to teaching schedules (timetables). Participants verbalised that self-management requires effective time-management, as indicated in the following participant statement: "I need to [take the] initiative and ensure that I am able to work within the required time so as to meet the deadline."

One participant indicated that by practicing self-leadership through self-management, nurse educators would be role modelling professional behaviours to students. The participant said:

"When you are a nurse educator you strive be a role model to students and you must be organised. Being organised means when you have a class at a certain time, you prepare lessons on time, develop well thought-out lesson plan[s] that are methodological ... in sequence."

"I manage my own time. How so? If there is content that needs to be prepared, I prepare it before going to class. And I grade students' assessments [before the] stipulated time. And then in class, I am guided by a timetable so that I do not go over the allocated teaching time. As a nurse educator, you know the time you are supposed to be on duty, the time you are supposed to leave. And then when it is time for you to do your job, you should do your job."

The abovementioned views attest to participants' recognition that practising self-leadership allows them to stay focused and in control of their responsibilities inside and outside of the classroom. Moreover, the participants view these practices as those that could motivate student learning and are worthy of student emulation. The participants indicated that their self-leadership entailed constantly asking themselves whether or not they were practicing or teaching relevantly in order to take the necessary steps to improve their own performance. As one participant stated: "I have to reflect on myself to look at the way I am performing, and reflect on whether I am performing as expected and then do something about that." One participant stated:

"You reflect on what you have done, what you can do better next time? Maybe you tried to achieve something that you realise then that I actually did not achieve what I wanted to, so 
you need to restructure that." Being a reflective practitioner also meant that participants had to monitor their behaviour and interactions with their colleagues and students. One participant stated:

"I must have the ability to reflect on myself-how I behave, how I talk, and quickly [implement] remedial actions if ever there is something that I think I am doing wrong."

The participants' views demonstrate that it is unnecessary for nurse educators to wait for external feedback or appraisal on their teaching. Rather, effective engagement with their self-leadership allows individuals to assess their strengths and weaknesses more accurately, and they can attempt to transform future actions and make meaningful changes.

The participants also indicated that being in possession of self-leadership means that nurse educators are likely to have the ability to acknowledge and accept any knowledge-deficit they might have. However, when such a deficit is identified, a nurse educator is expected to take the initiative and seek out knowledge by researching new information and identifying relevant sources, in order to be relevant in changing healthcare systems before going to class. The participants said the following:

"A nurse educator is expected to be knowledgeable to effectively facilitate knowledge to students. So, we are expected to take it upon ourselves to research and get accurate information on what you need to disseminate to the students."

"One strives to keep abreast with the latest development in the areas of interest, goes back and prepares some more, searches information, even from colleagues. One even asks them the type of questions students ask, and from there, those questions, you also will learn from your mistakes and improve your shortcomings."

The participants' views indicate the need for nurse educators to be reflective practitioners who yearn to continuously learn about the subject or topic they teach, so that they can assimilate the content and convey it in an understandable manner to students.

\subsubsection{Perception of self-leadership relating to students}

Nurse educators perceived their self-leadership in relation to their responsibilities towards their students. The participants' views indicate that nurse educators view self-leadership as an obligation to maintain professionalism by accepting responsibility and accountability towards students. As one participant stated: "We are teaching them to become independent registered nurses who take responsibility for their actions even in situations where they may not always have guidance."
The participants stated the following:

"Teaching gives me an idea of self-direction so that I carry along the responsibility and accountability to lead somebody to the future, the student."

"I look at self-leadership as an act of being responsible, being accountable towards students. One should be mindful that every decision made may have an impact on the students."

The participants' views demonstrate the respect that they have for their students and is an acknowledgement of the authority and influence that they have in fostering professionalism in their students. Nurse educators were aware that when they practiced self-leadership, students in turn developed and displayed behaviours consistent with professional standards.

\subsubsection{Perception of self-leadership within the community structure}

In this subtheme, participants perceived their self-leadership not only within the NEI, but also perceived self-leadership as contributing to the development of the community.

Nurse educators perceived themselves as having a responsibility or role to empower their communities and as well as learning from the community's experiences. For the participants, self-leadership meant taking initiatives in terms of working towards building on their contextualised knowledge of the community's culture and ensuring that their teaching practices are centred around the community's health needs. One participant stated:

"as a nurse educator, you have to immerse yourself in the community that you are living with or all types of people that are surrounding you. And you have to research about their life and their health needs. Learning doesn't only take place in an institution like a university; it happens even outside. The learning environment can be a university, at home, at the funeral, anywhere else."

Participants also described their self-leadership as an act of taking deliberate steps to support and empower local communities. One participant said:

"one of the biggest goals in my life is to make sure that those around me benefit from me, and I think the starting point here would be empowerment. My biggest goal is to be actively involved in the communities, but making sure that I empower other fellow women [by] going out there talking to them, especially on issues which are of national importance; gender-based problems, etc."

The participants' views cited above demonstrate the nurse educators' determination and motivation to be change agents in the communities in which they live or work.

ISSN 1925-4040 E-ISSN 1925-4059 


\subsection{Theme II: Facilitation of self-leadership in nurse ed-} ucators

In this theme, the participants described how nurse educators' self-leadership could be facilitated in an NEI. Both the nurse educators and the academic institution have a role to play in the facilitation of nurse educators.

\subsubsection{Self-facilitation of self-leadership}

In this subtheme, the participants suggested that individual educators themselves could facilitate nurse educators' selfleadership, through continuing professional development (CPD).

The participants viewed attainment of knowledge and skills through CPD as an intervention that would enhance their self-leadership. This view seemed to emanate from the participants' understanding that engagement in CPD enabled nurse educators to reflect and consider their attitudes and approaches to learning and teaching. One participant said: "I think as a nurse educator, you need to attend courses, which are relevant to the content that you are teaching, so that you can develop your teaching."

Participants stated examples of initiatives that individual nurse educators could attend to promote their self-leadership:

"I get my motivation from knowledge, like going to seminars. That makes you positive. Going to seminars, doing short courses, and all of that, it keeps my mind-set positive and motivated and broadens it."

"In order to be up-to-date with the content, one should always attend in-service trainings because they are there. We should always see to it that we get good development as lecturers ... all that, by so doing, we are always updating ourselves."

From the participants' sentiments, it is evident that as a selfdevelopmental initiative, CPD requires more effort from the individual nurse educator rather than the institution within which they work, and could assist in improving the learning quality and teaching process.

\subsubsection{Institutional-facilitation of self-leadership}

The participants suggested that self-leadership in nurse educators could also be facilitated by the organisation or institution in which they worked. The institution can facilitate nurse educator self-leadership through offering mentoring; succession planning; and management and collegial support.

The participants indicated that being mentored by experienced nurse educators and leaders within the institutions could facilitate their self-leadership. They identified mentorship as a missing institutional strategy, and as something that had to be demanded at times, as they longed for direct guidance, especially for newly employed nurse educators.

Published by Sciedu Press
Participants believed that mentoring by those that they admired, could enhance their self-leadership skills, as stated by one participant: "I believe that we would really like to see role models that will also guide us to ... bring out the best in us."

Another participant said:

"I think we need to be mentored, I have realised that what is lacking for us young nurses-or educators, is that we don't have that direct guidance. So, I would say if we had mentors, then we would develop into self-leaders."

The quotations above indicate that participants consider supportive interventions such as mentoring as a tool that could enhance nurse educators' self-leadership. This could be because mentoring provides structured guidance through institutional role models to improve nurse educators' teaching skills.

The participants suggested that the institution practice of succession planning could empower nurse educators to be self-leaders who could lead the institution in the future. One participant stated:

"The management of the institution should institute succession planning.... By succession planning, I mean that the least junior personnel in the institution must also be given the opportunity of leading the institution."

Some of the participants held the view that they were ready to transition from their current roles of being nurse educators to taking on the responsibilities of institutional management. One participant stated:

"The succession plan should be there so that when leaders leave positions, I should be prepared as somebody who is also a leader to be able to lead the institution." The statements above indicate that the participants believe that nurse educators' self-leadership could be facilitated if the institution implemented initiatives that deliberately expose nurse educators to institutional management positions. The participants were also of the view that their self-leadership could be facilitated by institutional management support. They expect management to foster an environment in which participants feel recognised, respected, and treated fairly, especially when nurse educators experience difficult situations. One of the participants said: "Even if it is a reward, the management can just say 'We are aware that you have been performing well, and we recognise and appreciate your work."' One participant indicated that involving nurse educators in decisionmaking processes could support them: "Give us opportunities to give inputs; don't underestimate our potential."

Some participants stated that the type of support they sought 
from the institutional management was related to their wellness. One participant said:

"If our seniors could provide support emotionally and psychologically, we can be stable at work. Because sometimes if you are having some problems, you feel like if I cannot go to work, I do not want to face my colleagues in this state."

The participants described supportive management as managers ensuring that there were adequate resources for learning and teaching in the NEI. One participant stated: "Some situations can be demotivating. You may find that a simulation laboratory does not have the necessary equipment to use when demonstrating to students."

Participants also lamented the lack of collegial support, which made participants feel demotivated and emotionally drained, which rendered them incapable of effectively engaging in learning and teaching activities. The lack of support led to nurse educators resorting to work in isolation, and it undermined collaboration and teamwork. A participant said: "Sometimes there is no support from peers, you know... There is no teamwork, in other words. There is serious isolation, there is no teamwork."

The quotations above attest to participants stating that in order for nurse educators' self-leadership to thrive, institutional leadership needs to provide educator support so that they feel valued within the institution.

\section{DisCussion}

The study established that nurse educators mirrored themselves as leaders in action and are aware of the importance of their engagement in self-leadership activities to learning and teaching. The nurse educators' perceptions of their own selfleadership were not solely confined within the NEIs within which they worked, but they also perceived themselves as community teachers who are relevant, responsive, and who contribute to the development of the community in which they lived. The facilitation of nurse educators' self-leadership was considered to be a shared responsibility between individual nurse educators and, to a certain extent, their colleagues, and institutional management.

\subsection{Perception of self-leadership}

This study found that participants perceived nurse educator self-leadership as a phenomenon that they were already practicing to a significant extent. In those areas in which they were unable to apply the concept, they referred educators' actions that they emulated, and referred to them as their role models. This awareness prompted the nurse educators to constantly monitor their thoughts and behaviours, as they were aware that students considered them to be their role models. Thus, when nurse educators practice self-leadership, they demonstrate commitment, respect, and responsibility towards learning and teaching students, colleagues, and the community. ${ }^{[21]}$ As leaders in their own right, nurse educators are valuable assets in their respective institutions, and as such, the expectation is that they will mirror the institution's core values in all the diverse and multiple tasks in which they engage. ${ }^{[12]}$

These findings support previous studies ${ }^{[22,23]}$ that focused on students' role-modelling in nursing education. Student nurses value educators who role-model by demonstrating knowledge and skills, possess good interpersonal skills, are well-prepared and enthusiastic, and value and respect their students. ${ }^{[2]}$ Such exemplary role-modelling affirms the students' values and improves their self-worth and confidence during learning and teaching. ${ }^{[22]}$

Nurse educators are influential role models in producing and training South African nurses. They are role-models to students and are also influential leaders in their communities. Thus it is imperative that nurse educators are self-leaders. Individuals with self-leadership possess elevated internal motivation, have higher personal expectations, and persist through difficulties, until they achieve their goals. ${ }^{[24]}$

\subsection{Self-leadership facilitative factors}

The study established that self-development initiatives such as CPD promotes self-leadership in nurse educators. This is due to the CPD potential that enhances educators' knowledge and skills, and enables them to consider their attitudes and approaches to learning and teaching. ${ }^{[25]}$ The CPD activities also enhance educators' abilities to self-reflect and self-evaluate, and facilitate attitudinal change during student and collegial interactions. ${ }^{[25]}$

Although self-leadership is considered a self-influencing or self-motivating project, self-leading nurse educators are part of a collegial community. Thus, the study found that even when individual nurse educators perceive themselves as selfmotivated, they also rely on external support to practice self-leadership. External support could take the form of institutional managers or educators' peers. Blackmore and Kandiko ${ }^{[26]}$ maintain that educators form part of a community of their peers with shared social practices, thinking, and discourse in their field, and whose "approval confers high intellectual standing".

Mentoring and succession planning were found to be valuable strategies that could be effective in improving nurse educators' performance in NEIs. Mentoring provides structured guidance to support new nurse educators, guiding them to perform job-required tasks, and in turn, mentees develop 
the skills required of an effective educator. These efforts lead to mentees being psychologically empowered, experiencing career satisfaction, self-confidence, and feelings that motivate personal growth, self-awareness, and self-reflection about their own educational practices. ${ }^{[27]}$

When facilitating self-leadership through succession planning, the NEI should identify nurse educators who are equipped to move into senior roles and begin training them immediately, so that they are ready to take on new opportunities within the institution. ${ }^{[28]}$

Supportive institutional managers create interpersonal, institutional, and financial conditions for nurse educators to become innovative and creative during learning and teaching. ${ }^{[2,30]}$ On the other hand, supportive educators make their colleagues feel accepted, a sense of belonging, and they consider themselves to be valuable to the institution. ${ }^{[31]}$ Thus, according to Fouché and colleagues, ${ }^{[31]}$ educators' caring and satisfying relationships with their peers lead to a sense of connectedness and improved performance.

\section{Study limitations}

The study provided rich results and discussions regarding the nurse educators' perceptions of their own self-leadership. The study was conducted in NEIs across only two of the nine South African provinces and therefore, the study findings cannot be generalised beyond these provinces, however they could be applied to other institutions in the remaining provinces. In spite of these limitations, the study provides insight into the nurse educators' descriptions of self-leadership and provide facilitative factors in an NEI context.

\section{Conclusion}

The nurse educators' perceptions provide a better understanding of nurse educators' views regarding self-leadership and self-leadership practice in nurse educators. The NEIs could benefit from assessing and supporting self-leadership practices. Training in self-leadership strategies could strengthen interpersonal skills amongst stakeholders in NEIs. Continuous self-reflection should be encouraged to ensure engagement in self-leadership practices during all activities in which nurse educators are involved, such as clinical accompaniment, clinical teaching, theory facilitation, institutional committees, teaching coordination, clinical learning, quality control, student administration, and student counselling.

Nursing skills are learnt through positive role modelling during students' clinical training facilitated by clinical preceptors and other professionals in the clinical areas. Thus, clinical preceptors should be trained in self-leadership skills, and always be mindful that students continually observe them so as not to underestimate the effect of their actions and behaviours on student nurses.

\section{CONFLicts OF INTEREST Disclosure}

The author declares that there is no conflict of interest.

\section{REFERENCES}

[1] South African Department of Health. Annual Performance Plan 2019/20-2021/22. Pretoria: Government Printers. 2019a.

[2] South African Department of Health. National Policy on Nursing Education and Training. Pretoria: Government Printers. 2019b.

[3] Blaauw D, Ditlopo P, Rispel LC. Nursing Education Reform in South Africa - Lessons From a Policy Analysis Study. Global Health Action. 2014; 7. PMid:25537941 https://doi .org/10.3402/gha.v7.2 6401

[4] South African Department of Public Service and Administration. Service Charter. PSCBC Resolution 1 of 2013. Pretoria: Public Service Co-ordinating Bargaining Council. 2013.

[5] South African Department of Public Service and Administration. Annual Performance Plan 2019/2020. Pretoria: DPSA. 2019.

[6] Clark C. Creating and Sustaining Civility In Nursing Education. Indianapolis: Sigma Theta Tau International. 2013.

[7] Altan S, Lane JF, Dottin E. Using Habits of Mind, Intelligent Behaviors, and Educational Theories to Create a Conceptual Framework for Developing Effective Teaching Dispositions. Journal of Teacher Education. 2019; 70(2): 169-183. https://doi.org/10.1177/ 0022487117736024

Published by Sciedu Press
[8] Ozdemir G. The Effect of Teachers' Self-Leadership Perceptions on Job Satisfaction. International Journal of Society Researchers. 2020; 15: 13-33. https://doi.org/10.26466/opus. 666043

[9] Jooste K. A Collage for Self-Reflection: The Changing Faces of Nursing Leadership. 2011. Available from: http://hdl . handle .net/10210/4065 (Accessed 14 June 2021).

[10] Stewart GL, Courtright SH, Manz CC. Self-leadership: A Paradoxical Core of Organizational Behavior. Annual Review of Organizational Psychology and Organizational Behavior. 2019; 6(1): 47-67. https : //doi.org/10.1146/annurev-orgpsych-012218-015130

[11] Ross S. A Conceptual Model for Understanding the Process of SelfLeadership Development and Action-Steps to Promote Personal Leadership Development. Journal of Management Development. 2014; 33(4): 299-323. https://doi.org/10.1108/JMD-11-2012-0 147

[12] Mendoza HGP. Self-Leadership and Core Self-Evaluations: An Approach to a Personal Management Development Program. Journal of Institutional Research in South East Asia. 2017; 15(3): 73-85.

[13] Neck CP, Manz CC, Houghton JD. The Definitive Guide to Personal Excellence. 2nd ed. Thousand Oaks: SAGE. 2019.

[14] Ntshingila N, Downing C, Hastings-Tolsma M. A Concept Analysis of Self-Leadership: The "Bleeding Edge" in Nursing Lead- 
ership. Nursing Forum. 2021; 56(2): 404-412. PMid:33484177 https://doi.org/10.1111/nuf.12551

[15] Matahela VE. Guidelines for the facilitation of self-leadership in nurse educators. Pretoria: University of South Africa. 2019.

[16] Yadav M, Mishra SK. Self-leadership as a Tool for Enhancing Performance at Workplace. GIS Business. 2019; 14(6): 76-88. https://doi.org/10.26643/gis.v14i6.11628

[17] Creswell JW. Research Design. Qualitative, Quantitative, and Mixed Method Approaches. 4th ed. Los Angeles: SAGE. 2014.

[18] Nowell LS, Norris JM, White DE, et al. Thematic Analysis: Striving to Meet the Trustworthiness Criteria. International Journal of Qualitative Methods. 2017; 16(1): 1-13. https://doi.org/10.1177/ 1609406917733847

[19] Lincoln YS, Guba EG. Naturalistic Inquiry. California: SAGE. 1985.

[20] Brink H, Van der Walt C, Van Rensburg G. Fundamentals of Research Methodology for Healthcare Professionals. 3rd ed. Cape Town: Juta. 2012.

[21] Killen R. Teaching Strategies for Quality Teaching and Learning. Claremont: Juta. 2010.

[22] Jack K, Hamshire C, Chambers A. The Influence of Role Models in Undergraduate Nurse Education. Journal of Clinical Nursing. 2017; 26(23-24): 4707-4715. PMid:28334475 https ://doi.org/10.1 $111 /$ jocn. 13822

[23] Perry RN. Role Modeling Excellence in Clinical Nursing Practice. Nurse Education in Practice. 2009; 9(1): 36-44. PMid:18590978 https://doi.org/10.1016/j.nepr.2008.05.001

[24] Watson L. Self-Leadership: Becoming an Exceptional Leader. Radiologic Technology. 2006; 75(60): 457-470.
[25] Reddy BS. Self-Empowerment Through Continuous Professional Development. International Journal on Studies in English Language and Literature. 2014; 2(3): 79-81.

[26] Blackmore P, Kandiko CB. Motivation in Academic Life: A Prestige Economy. Research in Post-Compulsory Education. 2011; 16(4): 399-411. https:/doi.org/10.1080/13596748.2011.626971

[27] Nowell L, Norris JM, Mrklas K, et al. Mixed Methods Systematic Review Exploring Mentorship Outcomes in Nursing Academia. Journal of Advanced Nursing. 2017; 73(3): 527-544. PMid:27650412 https://doi.org/10.1111/jan.13152

[28] Hardy K. Self-Leadership as a Tool in Management Succession Planning: Planned Research Could Prove Valuable in Meeting the Challenges of Succession Planning and Management. The Public Manager. 2004; 33(3): 41-44.

[29] Gonzalez GM, Sutton M. The Role of Supportive Leaders at Top Performing Universities: Best Practice Lessons from American Institutions for Indonesian Higher Education. International Journal of Leadership and Change. 2013; 1(1): 5-13. Available from: http://digitalcommons.wku.edu/ijlc/vol1/iss1/4 (Accessed 10 April 2019).

[30] Rafferty AE, Griffin MA. Refining Individualized Consideration: Distinguishing Developmental Leadership and Supportive Leadership. Journal of Occupational and Organizational Psychology. 2006; 79(1): 37-61. https ://doi.org/10.1348/096317905X36731

[31] Fouché E, Rothmann S, Van der Vyver C. Antecedents and Outcomes of Meaningful Work among Schoolteachers. SA Journal of Industrial Psychology. 2017; 43(0): 1-10. https://doi.org/10.4102/sa jip.v43i0.1398 